\title{
Markov Model-Based Energy Efficiency Spectrum Sensing in Cognitive Radio Sensor Networks
}

\author{
Yan Jiao and Inwhee Joe \\ Department of Electronics and Computer Engineering, College of Engineering, Hanyang University, 17 Haengdang-dong, \\ Sungdong-gu, Seoul 133-791, Republic of Korea
}

Correspondence should be addressed to Inwhee Joe; iwjoe@hanyang.ac.kr

Received 7 November 2015; Accepted 14 January 2016

Academic Editor: Pantelis-Daniel Arapoglou

Copyright ( 2016 Y. Jiao and I. Joe. This is an open access article distributed under the Creative Commons Attribution License, which permits unrestricted use, distribution, and reproduction in any medium, provided the original work is properly cited.

Cognitive Radio Sensor Network (CRSN), incorporating cognitive radio capability in wireless sensor networks, is a new paradigm of the next-generation sensor network. Sensor nodes are usually battery powered and hence have strict energy constraints. As a result, energy efficiency is also a very critical problem in the CRSN. In this paper, we focus on energy consumption because of spectrum sensing. Furthermore, we present an adaptive spectrum sensing time interval strategy, in which SUs can adjust the next spectrum sensing time interval according to the current spectrum sensing results (namely, channel status). In order to find an optimal spectrum sensing time interval, we introduce the Markov model. Then, we establish a Markov model-based mathematical modeling for analyzing the relationship between spectrum sensing time interval and prior spectrum sensing results. Finally, numerical results demonstrate that the proposed strategy with dynamic adaptive spectrum sensing time interval exceeded listen before talk (LBT) strategy which is widely used for traditional wireless sensor networks.

\section{Introduction}

Wireless sensor networks (WSNs) [1] have tremendous applications in health monitoring, wildlife tracking, air pollution monitoring, and the use of it for gully pot monitoring in urban areas. Since it consists of resource-constrained sensor nodes, long network lifetime is one of the fundamental requirements in WSN. Moreover, one of the primary objectives of WSNs is to transmit a large amount of data timely and simultaneously, without using a large amount of network resources. To deal with such problems, a new sensor networking paradigm with the dynamic spectrum access scheme is involved, in which wireless sensor nodes are equipped with cognitive radio capability. This new sensor networking paradigm is known as Cognitive Radio Sensor Networks (CRSN) [2]. The main design principles and features of CRSNs are discussed openly in the literature [3-5]. There are many advantages of CRSN, such as efficient spectrum usage, good radio propagation, and flexible deployment property. However, similar to the traditional WSN, a CRSN consists of a large number of low-cost, low-power sensor nodes (namely, secondary user) that operate on limited battery energy. Furthermore, comparing to traditional WSN, the spectrum sensing process will cause more energy consumption. Above all, energy efficiency is an urgent problem in CRSN.

There are some studies which have started researching this issue. Hareesh and Singh proposed a new hybrid cooperative spectrum sensing technique which associates energy and eigenvalue based detectors to improve the energy efficiency of CRSN in [6]. In [7], Phuong and Kim proposed an intelligent power control scheme to address the communication requirement based on the interference model of cumulative interference from the entire cognitive industrial sensor networks. Liu et al. proposed a fast differential evolution algorithm to optimize the energy consumption and spectrum sensing performance jointly in [8]. In [9], Maleki et al. proposed a combined sleeping and censoring scheme as an energy-efficient spectrum sensing technique for CRSNs. However, all of these studiesoverlooked a fact that spectrum sensing time also brings tremendous influence on energy consumption in CRSN. References [10-15] have focused on 
the throughput maximization rather than energy efficiency of CRSN, although they consider spectrum sensing time. References [16-19] also consider a fixed spectrum sensing time interval, although they focus on energy efficiency issues.

Different from previous studies, in this paper, we focus on a dynamic spectrum sensing strategy which the next spectrum sensing time is adaptive and based on current spectrum sensing results. That is, spectrum sensing time interval is not fixed, according to the current sensing results. However, in practice, sensing results may be inaccurate due to imperfect sensing in CRSN. Hence, we adopt a Markov model to describe the state change of channel. And then, we have formulated the energy-efficient problem as that of estimating the state of the Markov model. Finally, utilizing Markov model-based state prediction, we derive thresholds on the spectrum sensing time interval for the action policy, which specifies the optimal action achieving minimum energy consumption.

The remainder of the paper is organized as follows. In Section 2, we give a description of the system. Section 3 analyzes the problem from the mathematical aspect and derives the mathematical formulated to solve the problem. Simulation results are given in Section 4 and finally we draw a conclusion in Section 5.

\section{System Model}

2.1. Network Architecture. We consider a CRSN, in which sensors with cognitive capacity work with the ISM frequency channel. In addition, we assume that PUs are TV bands and $\mathrm{SU}$ is a pair set of $\mathrm{SU}$ transmitter $\left(\mathrm{SU}_{t x}\right)$ and $\mathrm{SU}$ receiver $\left(\mathrm{SU}_{r x}\right)$. SUs can access TV bands when they detect the TV bands which are not used by PUs; on the contrary, SUs work with the ISM frequency channel. We also assume that SUs can access a set of $M$ licensed frequency channels which are mutually independent [20]. The set of channels is indexed as $\{1,2, \ldots, m\}$, and each of them is assumed to occupy a bandwidth $B_{c}$.

2.2. Channel State Model. Similar to [21], a Markov chain model is applied to model the state of each channel which is shown in Figure 1. $S_{m}(t)$ is the state of the channel $m$ in the slot $t$, where $S_{m}(t) \in\{1$ (idle), 0 (busy) $\} . P_{01}^{m}$ and $P_{11}^{m}$ are the probability of channel $m$ to transmit busy state to idle state and keep idle state, respectively. They are denoted as follows:

$$
\begin{aligned}
& P_{01}^{m}=\operatorname{Pr}\left\{S_{m}(t)=1 \mid S_{m}(t-1)=0\right\}, \\
& P_{11}^{m}=\operatorname{Pr}\left\{S_{m}(t)=1 \mid S_{m}(t-1)=1\right\} .
\end{aligned}
$$

2.3. Cognitive Radio User Operating Model. In our Model, time-slotted decentralized medium access control mechanism is adopted. Each time slot can be split up to three main subslots shown in Figure 2. At the beginning of each time slot, sensing period $\tau_{s}$ is reserved for identification of the available channel (idle channel). With respect to sensing result, an SU decides which channel to operate in switching period $\tau_{\text {handoff }}$. If the channel is already used by an SU on the previous slot which is identified as the available channel,

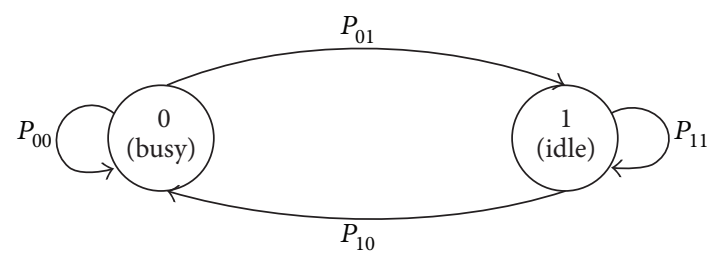

FIGURE 1: Channel state transition model.

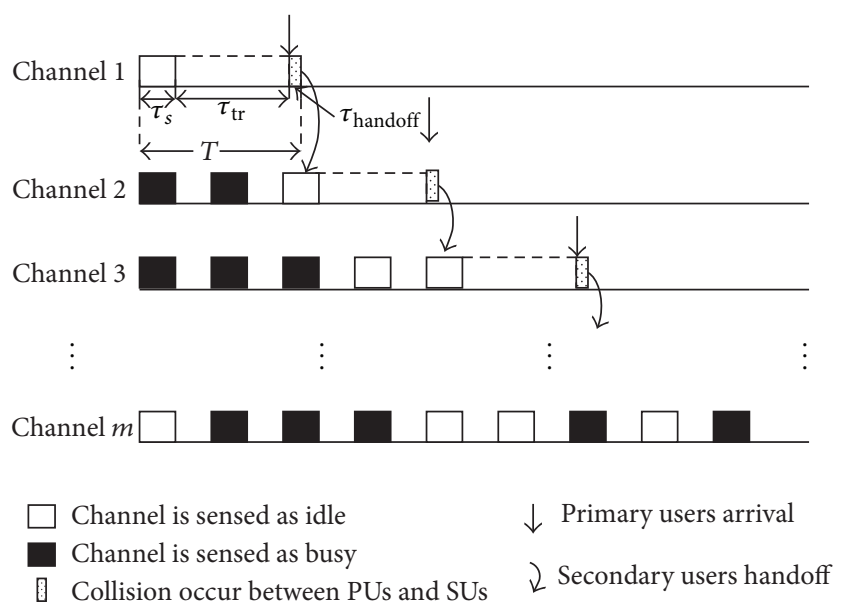

FIgure 2: General Dynamic Spectrum Access Scheme for CRSN.

the SU does not change its operating frequency and continues its transmission. Otherwise, an SU determines a new channel which must be defined as a spectrum hole at spectrum sensing time period. The actual transmission time of SU appears in the transmission period $\tau_{\mathrm{tr}}$. Furthermore, the SUs adopt the listen before talk strategy that SUs detect the channel of primary user in simple energy detection before they transmit the data; if the channel is sensed to be idle (state 1), the SUs transmit data. Otherwise, the channel is sensed to be busy (state 0 ), and the SUs must stop data transmission to keep waiting for the next result of next detection.

It has been shown that a sufficient statistic for optimal decision-making is given by the conditional probability that each channel is in state idle given all past decisions and observations [22]. The conditional idle state probability of each channel is denoted by $\Omega(t) \triangleq\left[\omega_{1}(t), \omega_{2}(t), \ldots, \omega_{c}(t)\right]$, namely, referred to as the belief vector, where $\omega_{c}(t)=\operatorname{Pr}\left\{S_{c}(t) \mid H_{c}(t)\right\}$ and $H_{c}(t)$ is all past decisions and observations in slot $t$. Therefore, the belief vector for slot $t+1$ can be obtained as given in

$$
\begin{aligned}
& \omega_{c}(t+1) \\
& = \begin{cases}P_{11} & a(t)=c, S_{a(t)}(t)=1 \\
P_{01} & a(t)=c, S_{a(t)}(t)=0 \\
\omega_{c}(t) P_{11}+\left(1-\omega_{c}(t)\right) P_{01} & a(t) \neq c,\end{cases}
\end{aligned}
$$


where $a(t)$ is given the sensing action denoted as $a(t) \epsilon$ $\{1,2, \ldots, C\}$ which shows selecting one channel $c$ by SUs.

Due to the imperfect sensing, the main indicators to measure the performance of spectrum sensing are detecting probability $P_{d}$, false alarm probability $P_{f}$. $P_{f}$ determines the percentage of the idle channel that is misclassified as occupied. When $P_{f}$ increase, it means that there is less opportunistic spectrum access. We adopt simple energy detection as the underlying detection scheme here. $P_{d}$ and $P_{f}$ are defined as follows:

$$
\begin{aligned}
& P_{d}\left(\lambda, \tau_{s}\right)=\operatorname{Pr}\left\{O\left(\tau_{s}\right)=0 \mid S_{c}\left(\tau_{s}\right)=0\right\}, \\
& P_{f}\left(\lambda, \tau_{s}\right)=\operatorname{Pr}\left\{O\left(\tau_{s}\right)=0 \mid S_{c}\left(\tau_{s}\right)=1\right\},
\end{aligned}
$$

where $\lambda$ denotes the sensing threshold of energy detector. In order to reduce interference to the primary user, $P_{d}$ is less than object detects probability $P_{d}^{*}$. According to [23], $P_{f}$ may be expressed as $P_{f}=Q\left(\sqrt{2 \gamma+1} Q^{-1}\left(P_{d}^{*}\right)+\sqrt{\tau_{s} f_{s}} \gamma\right)$, where $Q(x)$ is the Gaussian tail probability with inverse $Q^{-1}(\cdot) ; \gamma$ is the average received signal-to-noise rate (SNR) of PU's signal on each channel; $f_{s}$ represents the sampling frequency in hertz. Combining (2) and (3), we can obtain

$$
\begin{aligned}
& \omega_{c}(t+1)=\operatorname{Pr}\left\{S_{c}(t+1) \mid \Omega(t), a(t), S_{a(t)}\right\} \\
& = \begin{cases}P_{11}^{c} & a(t)=c, S_{a(t)}=1 \\
\frac{P_{f} \omega_{c}(t) P_{11}^{c}+\left(1-\omega_{c}(t)\right) P_{01}^{c}}{P_{f} \omega_{c}(t)+\left(1-\omega_{c}(t)\right)} & a(t)=c, S_{a(t)}=0 \\
P_{01}^{c}+\left(P_{11}^{c}-P_{01}^{c}\right) \omega_{i}(t) & a(t) \neq c .\end{cases}
\end{aligned}
$$

\section{Energy Consumption Analysis of System}

3.1. Energy Consumption Model. Energy dissipation by a sensor node can be attributed to transmitting and receiving data. When a transmitter transmits a unit of message to a receiver and the distance of them is denoted by $d$, so the energy consumption at the transmitter can be calculated as [24]. One has

$$
E_{t x}=\bar{E}_{r x, \min } \frac{(4 \pi d)^{\theta}}{g_{t x} g_{r x} \alpha^{2}} f^{2}
$$

where $\bar{E}_{r x, \min }$ is the minimum energy of receiving bit per until packet at the receiver with given modulated mode and error rate; $g_{t x}$ and $g_{r x}$ denote antenna gain of sender and receiver, respectively. Due to mostly sensor node with the omnidirectional antenna, values $g_{t x}$ and $g_{r x}$ are generally 1 ; $\alpha$ is the speed of propagation light; $f$ denotes the frequency of the carrier; the propagation loss factor $\theta$ typically varies between 2 and 4 .

3.2. Energy Consumption of SU. In our system model, as mentioned above, the SUs are probably in two states: without spectrum sensing and with spectrum sensing. The former means that the SUs can transmit data with enough spectrum resource (namely, ISM frequency) so that they need not sense idle spectrum unused by PUs; the latter means that SUs have not enough spectrum resource so that they need to sense idle spectrum unused by PUs to transmit data. Hence, we analyze two cases of energy consumption for SUs as follows.

3.2.1. The Energy Consumption of SU Node without Spectrum Sensing. If the SUs have enough spectrum resource to transmit data, so they need not sense spectrum. In this case, the energy consumption of CRSN like common WSN occurs in SUs transmitting phase, SUs receiving phase, and transmitting data with ISM frequency channel phase. Hence, we can obtain that the total energy consumption of $\kappa$ bits data transmission can be expressed as

$$
E_{1}=\kappa\left(E_{\mathrm{SU}_{t x}}+E_{\mathrm{ISM}}\right)+\kappa E_{\mathrm{SU}_{r x}}
$$

where $E_{\text {ISM }}$ is the energy consumption of 1 bit data transmitted by ISM channel. $E_{\mathrm{SU}_{t x}}$ and $E_{\mathrm{SU}_{r x}}$ denote the energy consumption of SU transmitter and receiver when 1 bit data are transferred between them.

3.2.2. The Energy Consumption of SU Node with Spectrum Sensing. If the SUs have not enough spectrum resource, they need to perform spectrum sensing and have an opportunity to use the idle channel without PUs. In this case, the data transmission between SU sender and SU receiver includes sensing phase, decision phase, switching phase, transmission phase, and receiving phase. If a channel is busy by sensing, SUs need not switch current ISM frequency channel to another channel. In this case, comparing the without spectrum sensing cases, more energy is only consumed in spectrum sensing and decision phase. Hence, the energy consumption of $\kappa$ bits packet transmission can be expressed as

$$
E_{\mathrm{busy}}=E_{\mathrm{sp}}+E_{d}+\kappa\left(E_{\mathrm{SU}_{t x}}+E_{\mathrm{ISM}}\right)+\kappa E_{\mathrm{SU}_{r x}},
$$

where $E_{\mathrm{sp}}$ denotes energy consumption of spectrum sensing and $E_{d}$ denotes energy consumption by making a decision by the SU. On the other hand, the channel is sensed idle; the SU will switch to such channel for transmitting data. Hence, extra energy consumption occurs in switching phase. The energy consumption of $\kappa$ bits data transmission can be rewritten as

$$
E_{\mathrm{idle}}=E_{\mathrm{sp}}+E_{d}+E_{h}+\kappa\left(E_{\mathrm{SU}_{t x}}+E_{\mathrm{sc}}\right)+\kappa E_{\mathrm{SU}_{r x}},
$$

where $E_{h}$ denotes energy consumption of SU switching from the ISM channel to sensing channel in the ideal state. $E_{\mathrm{sc}}$ is the energy consumption of data transmitted by the available sensing channel.

As above, the average of energy consumption of SU that accesses channel $c$ can be expressed as

$$
E_{\mathrm{ave}}^{c}=E_{\mathrm{idle}}^{c} \frac{P_{01}^{c}}{P_{01}^{c}+\left(1-P_{11}^{c}\right)}+E_{\mathrm{busy}}^{c} \frac{1-P_{11}^{c}}{P_{01}^{c}+\left(1-P_{11}^{c}\right)} .
$$


Substituting (4) into (9), we can obtain (9) which is rewritten as follows:

$$
\begin{aligned}
E_{\mathrm{ave}}^{c}= & E_{\mathrm{idle}}^{c} \frac{\left(P_{f} \omega_{c}(t) P_{11}^{c}+\left(1-\omega_{c}(t)\right) P_{01}^{c}\right) /\left(P_{f} \omega_{c}(t)+\left(1-\omega_{c}(t)\right)\right)}{\left(P_{f} \omega_{c}(t) P_{11}^{c}+\left(1-\omega_{c}(t)\right) P_{01}^{c}\right) /\left(P_{f} \omega_{c}(t)+\left(1-\omega_{c}(t)\right)\right)+\left(1-P_{11}^{c}\right)} \\
& +E_{\mathrm{busy}}^{c} \frac{1-P_{11}^{c}}{\left(P_{f} \omega_{c}(t) P_{11}^{c}+\left(1-\omega_{c}(t)\right) P_{01}^{c}\right) /\left(P_{f} \omega_{c}(t)+\left(1-\omega_{c}(t)\right)\right)+\left(1-P_{11}^{c}\right)}
\end{aligned}
$$

To simply formula, we use $X, Y$, and $\beta$ which denote $P_{01}^{c}, P_{11}^{c}$, and $\omega_{c}(t)$, respectively. So we obtain (9) which can be shown as

$$
\begin{aligned}
E_{\text {ave }}^{c}= & E_{\text {idle }}^{c} \frac{Y \cdot\left[P_{f} \cdot \beta+(1-\beta)\right]}{P_{f} \cdot \beta+(1+X-Y) \cdot(1-\beta)} \\
& +E_{\text {busy }}^{c} \frac{(1-Y) \cdot\left[P_{f} \cdot \beta+(1-\beta)\right]}{P_{f} \cdot \beta+(1+X-Y) \cdot(1-\beta)} .
\end{aligned}
$$

3.3. Energy Efficiency Based on Markov Model. We assume that $\tau_{0}$ is an interval of next spectrum sensing when current sensing result is busy. Hence, the total energy consumption of $\tau_{0}$ is shown as

$$
E_{\tau_{0}}=E_{\text {busy }}+\tau_{0} E_{1} .
$$

We assume that $\tau_{1}$ is an interval of next spectrum sensing when current sensing result is idle. The total energy consumption between current idle state and next spectrum sensing starting is shown as

$$
E_{\tau_{1}}=E_{\text {idle }}+\tau_{1} E_{1} .
$$

We assume that $A$ is transition probabilities metric, which can be denoted by

$$
\begin{aligned}
A & =\left(a_{i j}\right)_{K \times K}, \\
a_{i j} & =\operatorname{Pr}\left(X_{\tau_{s}+1}=s_{j} \mid X_{\tau_{s}}=s_{i}\right), \quad i, j=1, \ldots, K,
\end{aligned}
$$

where $a_{i j}$ is the transition probability of the current state $s_{i}$ to the next state $s_{j} T_{s}=1,2,3, \ldots, T$ which is spectrum sensing time, $K$ is the number of states ( $K=2$ in this paper), and $X_{\tau_{s}} \in\left\{s_{1}, s_{2}, \ldots, s_{K}\right\},\left\{s_{1}, s_{2}, \ldots, s_{K}\right\}$ is hidden state space. Hence, the transition probability matrixes $A$ and $(A)^{n}$ can be written as, respectively,

$$
\begin{aligned}
A= & \left(\begin{array}{ll}
a_{00} & a_{01} \\
a_{10} & a_{11}
\end{array}\right)=\left(\begin{array}{ll}
P_{00} & P_{01} \\
P_{10} & P_{11}
\end{array}\right), \\
(A)^{n}= & \left(\begin{array}{ll}
a_{00}(n) & a_{01}(n) \\
a_{10}(n) & a_{11}(n)
\end{array}\right)=\left(\begin{array}{ll}
a_{00} & a_{01} \\
a_{10} & a_{11}
\end{array}\right)^{n} \\
= & \frac{1}{P_{01}+P_{11}}\left(\begin{array}{ll}
P_{11} & P_{01} \\
P_{11} & P_{01}
\end{array}\right) \\
& +\frac{\left(1-P_{01}-P_{11}\right)^{n}}{P_{01}+P_{11}}\left(\begin{array}{cc}
P_{01} & -P_{01} \\
-P_{11} & P_{11}
\end{array}\right),
\end{aligned}
$$

where $a_{i j}(n)(i, j \in 0,1)$ means that starting in state $s_{i}$ will be in state $s_{j}$ after $n$ steps.

We assume that $P_{\text {busy }}\left(\tau_{0}, \tau_{1}\right)$ and $P_{\text {idle }}\left(\tau_{0}, \tau_{1}\right)$ are the probability of sensing result, busy and idle, respectively. We can also come to a conclusion based on queuing theory:

$$
\begin{aligned}
P_{\text {busy }}\left(\tau_{0}, \tau_{1}\right) & =\frac{a_{10}\left(\tau_{1}\right)}{a_{10}\left(\tau_{1}\right)+a_{01}\left(\tau_{1}\right)} \\
& =\frac{P_{10}\left(\tau_{1}\right)}{P_{10}\left(\tau_{1}\right)+P_{01}\left(\tau_{1}\right)}, \\
P_{\text {idle }}\left(\tau_{0}, \tau_{1}\right) & =\frac{a_{01}\left(\tau_{0}\right)}{a_{01}\left(\tau_{0}\right)+a_{10}\left(\tau_{1}\right)} \\
& =\frac{P_{01}\left(\tau_{0}\right)}{P_{01}\left(\tau_{0}\right)+P_{10}\left(\tau_{1}\right)} .
\end{aligned}
$$

Combining (12) and (15), we can obtain total energy consumption as shown as follows:

$$
\begin{aligned}
E_{\text {tot }} & =P_{\text {busy }}\left(\tau_{0}, \tau_{1}\right) E_{\tau_{0}}+P_{\text {idle }}\left(\tau_{0}, \tau_{1}\right) E_{\tau_{1}} \\
& =\frac{P_{10}\left(\tau_{1}\right)}{P_{10}\left(\tau_{1}\right)+P_{01}\left(\tau_{0}\right)} E_{\tau_{0}}+\frac{P_{01}\left(\tau_{0}\right)}{P_{01}\left(\tau_{0}\right)+P_{10}\left(\tau_{1}\right)} E_{\tau_{1}} .
\end{aligned}
$$

The average of the total consumed energy in unit slot can be written as

$$
E_{\mathrm{ave}}=\frac{E_{\mathrm{tot}}}{T} \Longrightarrow E_{\mathrm{ave}}^{c}=\frac{E_{\mathrm{tot}}^{c}}{T} .
$$

Hence, the problem of exchange to the optimization problem is written as follows:

$$
\begin{array}{ll}
\min _{\tau_{0}, \tau_{1}} & E_{\text {ave }}^{c} \\
\text { s.t. } & \tau_{0}, \tau_{1} \in Z^{+} .
\end{array}
$$

Equation (19) can be rewritten as (20), and the derivation process is shown in the appendix. One has

$$
\begin{array}{ll}
\min _{\tau_{0}, \tau_{1}} & E_{\text {ave }} \\
\text { s.t. } & \tau_{0} \in\left[1, \tau_{0 \max }\right], \tau_{1} \in\left[1, \tau_{1 \max }\right] .
\end{array}
$$

According to the above-mentioned, when the optimal solution is $\left(\tau_{0 \max }, \tau_{1 \max }\right)$, the CRS stop sensing. Otherwise, according to $\left(\tau_{0 \text { opt }}, \tau_{1 \text { opt }}\right)$, the sensing result is busy (idle), waiting for $\tau_{0 \text { opt }}\left(\tau_{1 \text { opt }}\right)$ and then sensing again. 


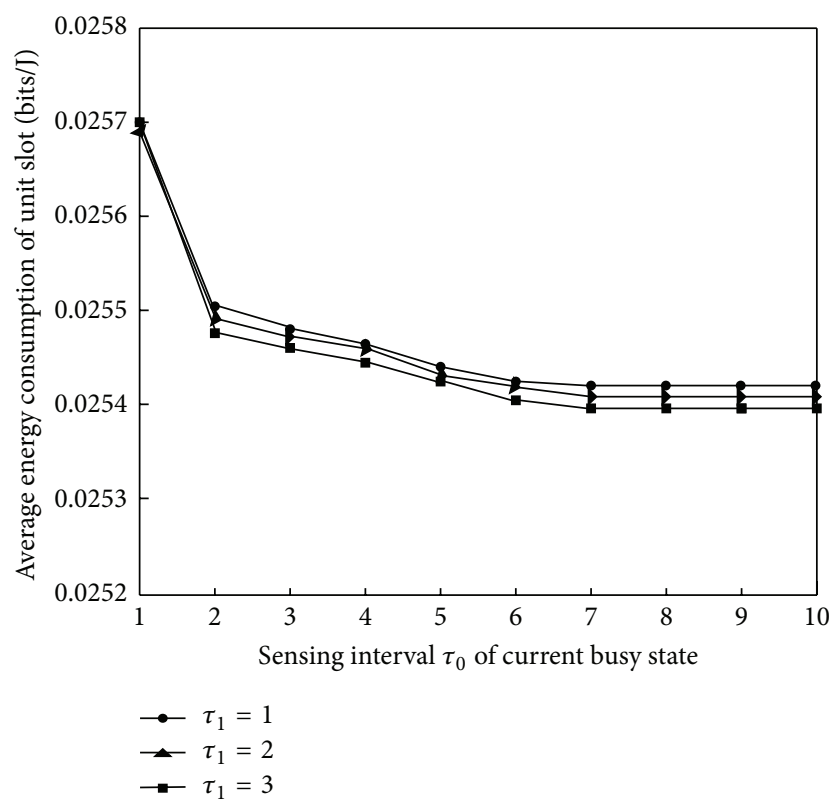

(a)

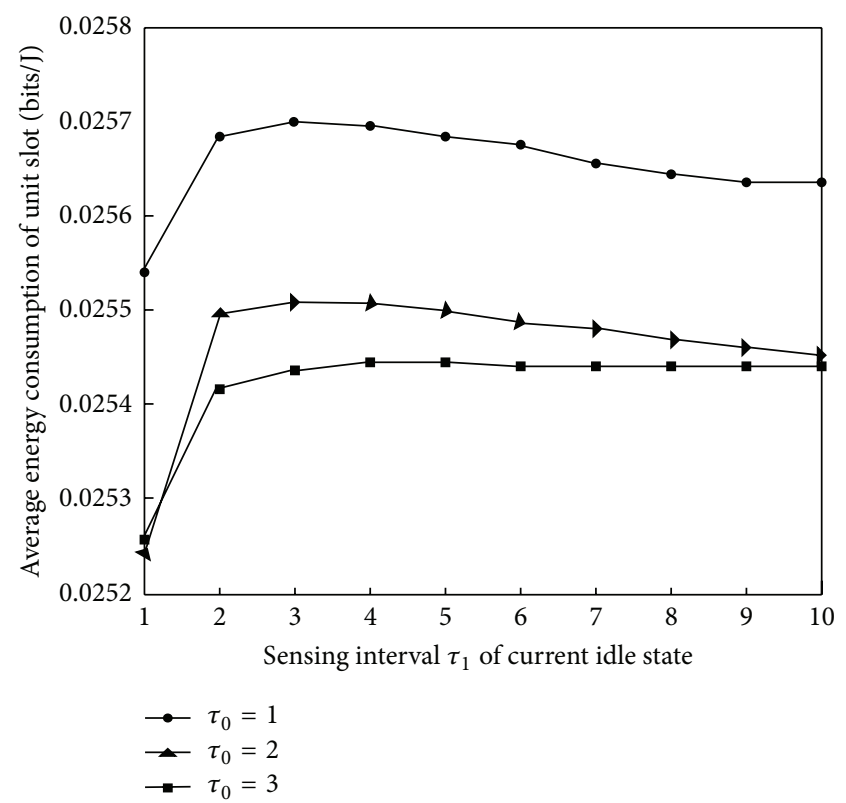

(b)

FIGURE 3: Average energy consumption of different time interval in a unit slot.

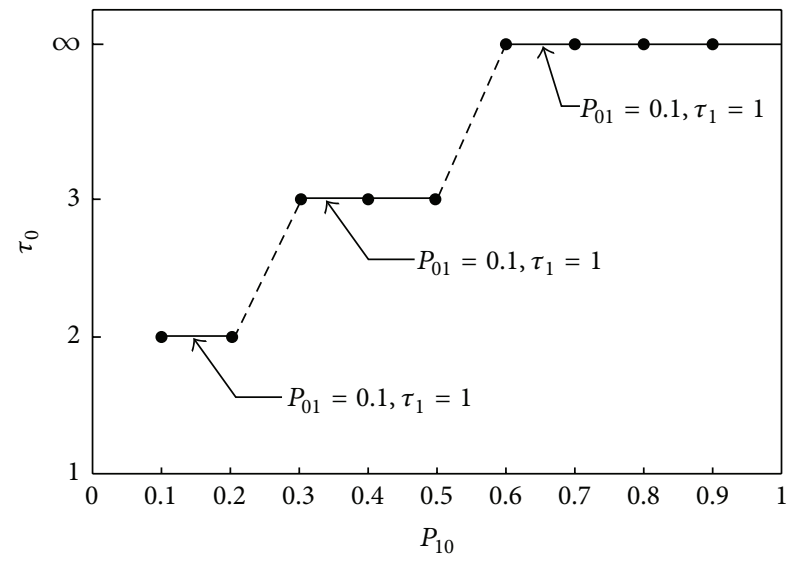

(a)

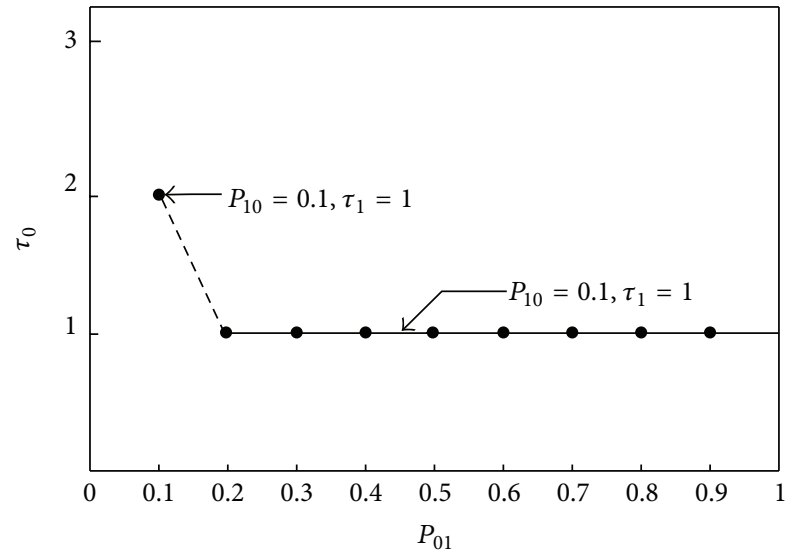

(b)

FIgURE 4: Optimal interval of combination $\left(\tau_{0}, \tau_{1}\right)$ with different $\left(P_{01}, P_{10}\right)$.

\section{Simulation}

In this section, we run Monte-Carlo simulations to find optimal next spectrum interval based on current spectrum results. In all simulations, we assume the parameters $P_{01}=$ $0.1, P_{10}=0.5, \bar{E}_{b}=1 \mathrm{pJ} / \mathrm{bit}, d=50 \mathrm{~m}, r=2, f=200 \mathrm{MHz}$, $f_{\mathrm{ISM}}=2.4 \mathrm{GHz}, \kappa=1000 \mathrm{bit}, E_{\mathrm{SU}_{\mathrm{tx}}}=50 \mathrm{~nJ} / \mathrm{bit}, E_{\mathrm{SU}}=$ $50 \mathrm{~nJ} / \mathrm{bit}, E_{h}=1 \mathrm{~mJ}$, and $E_{\mathrm{sc}}=20 \mathrm{~mJ}$.

Figure 3 demonstrates average energy consumption of $\tau_{0}\left(\tau_{1}\right)$ in a unit slot. From Figure 3 we can find that there is an optimal solution with a certain time interval, such that $\tau_{0}\left(\tau_{1}\right)$ value is 3 . It is also verifying our idea of reduction of energy consumption through spectrum sensing with a certain time interval.

Figure 4 shows an optimal interval of combination $\left(\tau_{0}, \tau_{1}\right)$ with different $\left(P_{01}, P_{10}\right)$. It demonstrates that the optimal interval can be found in small probability space. That is, searching optimal interval is available in limited space. However, interval $\tau_{0}$ approaches infinite when $P_{10}$ increase because $P_{10}$ increase means the channel in busy state with high probability. That means it is more energy-efficient without spectrum sensing.

Figure 5 shows average consumption in a unit slot with a different combination of $\left(\tau_{0}, \tau_{1}\right)$. The simulation is nearly 


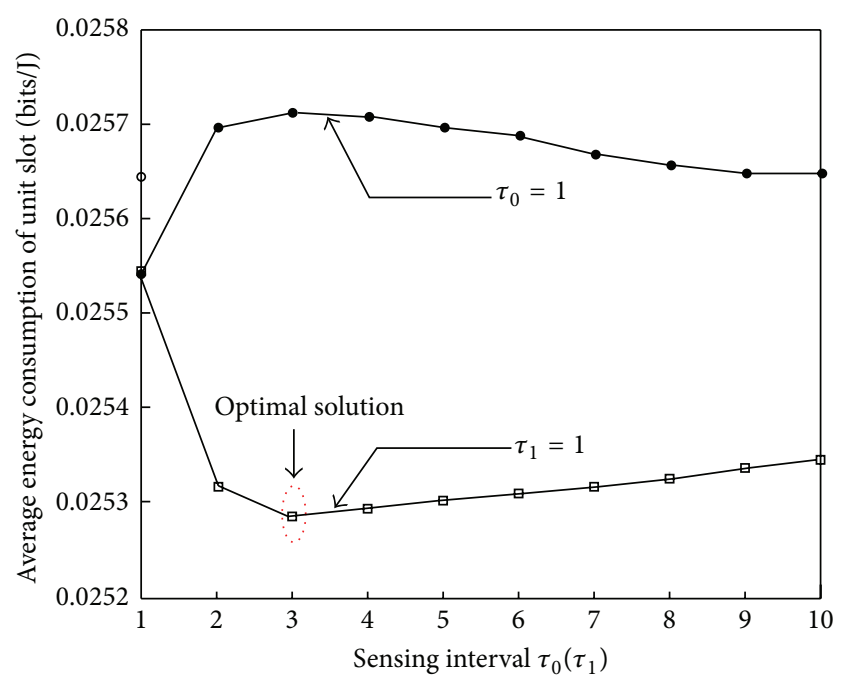

FIgURE 5: Average consumption in a unit slot with different combination of $\left(\tau_{0}, \tau_{1}\right)$.

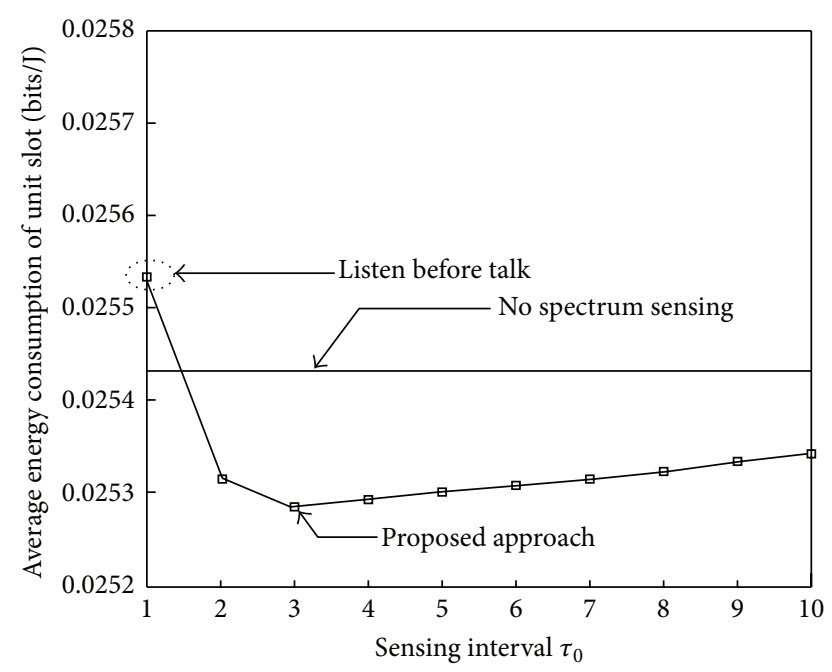

Figure 6: Comparison between "listen before talk" approach and without spectrum sensing approach.

identical to the theoretical value and also demonstrates the validity of (20).

The comparison between our proposed approach, "listen before talk" approach, and without spectrum sensing approach is shown in Figure 6. From Figure 6, we can find that comparing "listen before talk" and no spectrum sensing, our proposed approach is more efficient. Particularly, sensing time interval achieves an optimal value.

Figure 7 shows that our proposed approach is better than the approach referred to in [16]. From Figure 7, we can find in our proposed approach that the sensing time is longer and the energy efficiency is more effective, since our proposed approach can adapt sensing time starting according to

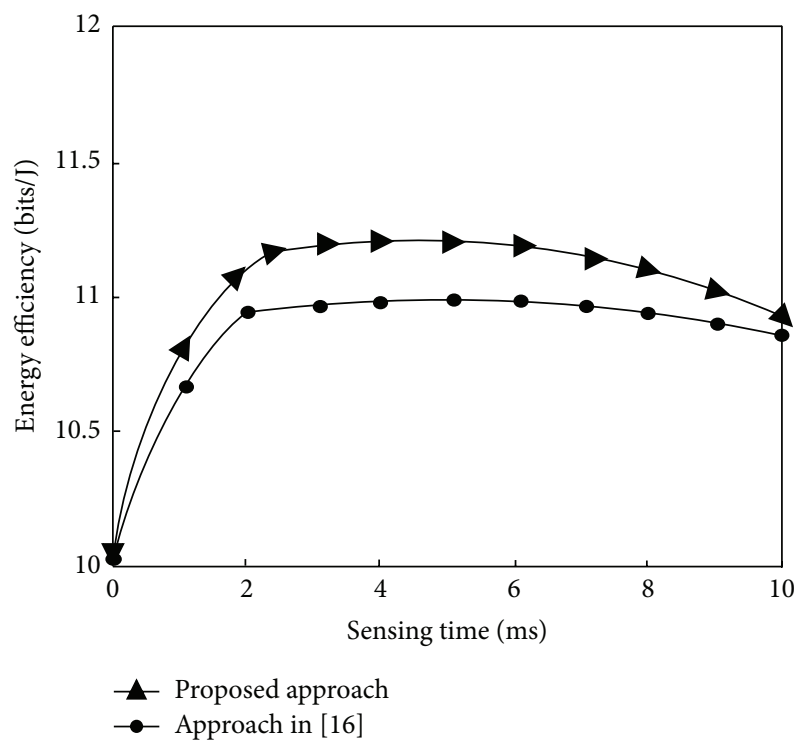

FIGURE 7: Comparison between proposed approach and approach in [16].

the prior sensing results. That is, our proposed approach does not perform spectrum sensing if the channel is busy and then reduces spectrum sensing energy consumption and improves the energy efficiency.

\section{Conclusion}

We proposed a study using Markov model with state prediction for spectrum sensing in CRSN. There is an optimal spectrum sensing interval which reduces total energy consumption. Simulation results show that, due to nonfixed sensing time interval, the prediction scheme may reduce energy consumption. This suggests that using the precision scheme to obtain an optimal spectrum sensing time interval can alleviate the energy consumption to improve the energy efficiency of CRSN.

\section{Appendix}

\section{The Derivation Process of (19)}

We rewrite (19) as follows:

$$
\left|E_{\text {ave }}^{c}\right|=\left|\frac{E_{\text {tot }}^{c}}{T}\right|
$$

because $0 \prec P_{10} \prec 1,0 \prec P_{01} \prec 1$ and based on (16) and (8), $\left|E_{\text {ave }}^{c}\right|$ is computed as follows:

$$
\left|E_{\text {ave }}^{c}\right| \leq\left|\frac{\left(E_{\text {busy }}-E_{\tau_{1}}\right)+\left(E_{\text {idle }}-E_{\tau_{1}}\right)}{P_{10}\left(\tau_{1}\right) \tau_{0}+P_{01}\left(\tau_{0}\right) \tau_{1}}+E_{\tau_{1}}\right| .
$$


We can rewrite this inequality as follows:

$$
\begin{gathered}
\left|E_{\text {idle }}^{c} \frac{P_{01}^{c}}{P_{01}^{c}+\left(1-P_{11}^{c}\right)}+E_{\text {busy }}^{c} \frac{1-P_{11}^{c}}{P_{01}^{c}+\left(1-P_{11}^{c}\right)}\right| \\
\leq\left|\frac{\left(E_{\text {busy }}-E_{\tau_{1}}\right)+\left(E_{\text {idle }}-E_{\tau_{1}}\right)}{P_{10}\left(\tau_{1}\right) \tau_{0}+P_{01}\left(\tau_{0}\right) \tau_{1}}+E_{\tau_{1}}\right| .
\end{gathered}
$$

And we further rewrite this inequality as follows:

$$
\begin{aligned}
& \left|E_{\text {idle }}^{c} \frac{P_{01}^{c}}{P_{01}^{c}+\left(1-P_{11}^{c}\right)}+E_{\text {busy }}^{c} \frac{1-P_{11}^{c}}{P_{01}^{c}+\left(1-P_{11}^{c}\right)}\right| \\
& \quad \leq \frac{\left|E_{\text {busy }}-E_{\tau_{1}}\right|+\left|E_{\tau_{1}}-E_{\text {idle }}\right|}{P_{01}\left(\tau_{0}\right) \tau_{1}}+E_{\tau_{1}} \\
& \quad \leq \frac{\left|E_{\text {busy }}-E_{\tau_{1}}\right|+\left|E_{\tau_{1}}-E_{\text {idle }}\right|}{P_{01} \min \tau_{1}}+E_{\tau_{1}},
\end{aligned}
$$

where $P_{0 \min }=\min \left(\left(P_{01}\left(\tau_{0}\right)\right), \tau_{0} \in[1, \infty]\right),\left|E_{\text {ave }}\right|>0$. Hence, we can denote $\tau_{1 \max }=\left(E_{\text {busy }}-E_{\text {idle }}\right) / P_{0 \min } \mu E_{\tau_{1}}$, where $\mu$ is correction parameter.

Similarly, we can denote $P_{1 \min }=\min \left(\left(P_{10}\left(\tau_{1}\right)\right), \tau_{1} \in\right.$ $[1, \infty])$ and $\tau_{0 \max }=\left(E_{\text {busy }}-E_{\text {idle }}\right) / P_{1 \min } \mu E_{\tau_{1}}$.

\section{Conflict of Interests}

The authors declare that there is no conflict of interests regarding the publication of this paper.

\section{References}

[1] W. B. Heinzelman, A. P. Chandrakasan, and H. Balakrishnan, "An application-specific protocol architecture for wireless microsensor networks," IEEE Transactions on Wireless Communications, vol. 1, no. 4, pp. 660-670, 2002.

[2] O. B. Akan, O. B. Karli, and O. Ergul, "Cognitive radio sensor networks," IEEE Network, vol. 23, no. 4, pp. 34-40, 2009.

[3] A. S. Zahmati, S. Hussain, X. Fernando, and A. Grami, "Cognitive wireless sensor networks: emerging topics and recent challenges," in Proceedings of the IEEE Toronto International Conference Science and Technology for Humanity (TIC-STH '09), pp. 593-596, IEEE, Toronto, Canada, September 2009.

[4] H. G. Goh, K. H. Kwong, C. Shen, C. Michie, and I. Andonovic, "CogSeNet: a concept of cognitive wireless sensor network," in Proceedings of the 7th IEEE Consumer Communications and Networking Conference (CCNC '10), pp. 1-2, Las Vegas, Nev, USA, January 2010.

[5] G. Vijay, E. Bdira, and M. Ibnkahla, "Cognitive approaches in wireless sensor networks: a survey," in Proceedings of the 25th Queen's Biennial Symposium on Communications (QBSC '10), pp. 177-180, Ontario, Canada, May 2010.

[6] K. Hareesh and P. Singh, "An energy efficient hybrid cooperative spectrum sensing technique for CRSN," in Proceedings of the IEEE International Multi Conference on Automation, Computing, Control, Communication and Compressed Sensing (iMac4s '13), pp. 438-442, IEEE, Kottayam, India, March 2013.

[7] T. M. Phuong and D.-S. Kim, "Efficient power control scheme for cognitive industrial sensor networks," International Journal of Control and Automation, vol. 7, no. 3, pp. 177-188, 2014.
[8] W. Liu, G. Qin, S. Li, J. He, and X. Zhang, "A multiobjective evolutionary algorithm for energy-efficient cooperative spectrum sensing in cognitive radio sensor network," International Journal of Distributed Sensor Networks, vol. 2015, Article ID 581589, 13 pages, 2015.

[9] S. Maleki, G. Leus, S. Chatzinotas, and B. Ottersten, “To AND or To OR: on energy-efficient distributed spectrum sensing with combined censoring and sleeping," IEEE Transactions on Wireless Communications, vol. 14, no. 18, pp. 4508-4521, 2015.

[10] Y.-C. Liang, Y. Zeng, E. C. Y. Peh, and A. T. Hoang, "Sensingthroughput tradeoff for cognitive radio networks," IEEE Transactions on Wireless Communications, vol. 7, no. 4, pp. 1326-1337, 2008.

[11] D.-J. Lee and M.-S. Jang, "Optimal spectrum sensing time considering spectrum handoff due to false alarm in cognitive radio networks," IEEE Communications Letters, vol. 13, no. 12, pp. 899-901, 2009.

[12] J. He, C. Xu, and L. Li, "Joint optimization of sensing time and decision thresholds for wideband cognitive OFDM radio networks," in Proceedings of the IET 3rd International Conference on Wireless, Mobile and Multimedia Networks (ICWMMN '10), pp. 230-233, September 2010.

[13] X. Zhang, Q. Wu, and J. Wang, "Optimization of sensing time in multichannel sequential sensing for cognitive radio," International Journal of Communication Systems, vol. 26, no. 2, pp. 222-235, 2013.

[14] O. van den Biggelaar, J.-M. Dricot, P. D. Doncker, and F. Horlin, "Sensing time and power allocation for cognitive radios using distributed Q-learning," EURASIP Journal on Wireless Communications and Networking, vol. 2012, article 138, 40 pages, 2012.

[15] H. Shokri-Ghadikolaei and R. Fallahi, "Intelligent sensing matrix setting in cognitive radio networks," IEEE Communications Letters, vol. 16, no. 11, pp. 1824-1827, 2012.

[16] Y. Pei, Y.-C. Liang, K. C. Teh, and K. H. Li, "Energy-efficient design of sequential channel sensing in cognitive radio networks: optimal sensing strategy, power allocation, and sensing order," IEEE Journal on Selected Areas in Communications, vol. 29, no. 8, pp. 1648-1659, 2011.

[17] S. Wang, Y. Wang, J. P. Coon, and A. Doufexi, "Energy-efficient spectrum sensing and access for cognitive radio networks," IEEE Transactions on Vehicular Technology, vol. 61, no. 2, pp. 906-912, 2012.

[18] S. Maleki, S. P. Chepuri, and G. Leus, "Optimization of hard fusion based spectrum sensing for energy-constrained cognitive radio networks," Physical Communication, vol. 9, pp. 193198, 2013.

[19] S. Maleki and G. Leus, "Censored truncated sequential spectrum sensing for cognitive radio networks," IEEE Journal on Selected Areas in Communications, vol. 31, no. 3, pp. 364-378, 2013.

[20] Y. Cheng, H. Li, and P.-J. Wan, "A theoretical framework for optimal cooperative networking in multiradio multichannel wireless networks," IEEE Wireless Communications, vol. 19, no. 2, pp. 66-73, 2012.

[21] X. Ma, S. M. Djouadi, and H. S. Li, "State estimation over a semiMarkov model based cognitive radio system," IEEE Transactions on Wireless Communications, vol. 11, no. 7, pp. 2391-2401, 2012.

[22] Q. Zhao, B. Krishnamachari, and K. Q. Liu, "On myopic sensing for multi-channel opportunistic access: structure, optimality, and performance," IEEE Transactions on Wireless Communications, vol. 7, no. 12, pp. 5431-5440, 2008. 
[23] M. C. Oto and O. B. Akan, "Energy-efficient packet size optimization for cognitive radio sensor networks," IEEE Transactions on Wireless Communications, vol. 11, no. 4, pp. 15441553, 2012.

[24] A. S. K. Mammu, A. Sharma, U. Hernandez-Jayo, and N. Sainz, "A novel cluster-based energy efficient routing in wireless sensor networks," in Proceedings of the 27th IEEE International Conference on Advanced Information Networking and Applications (AINA '13), pp. 41-47, Barcelona, Spain, March 2013. 


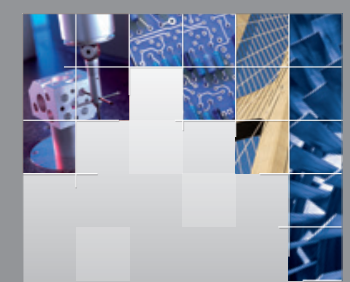

\section{Enfincering}
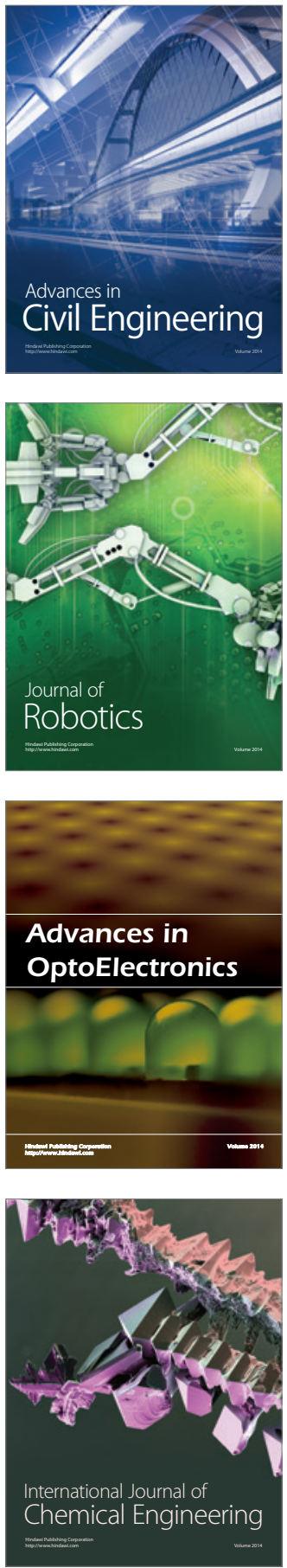

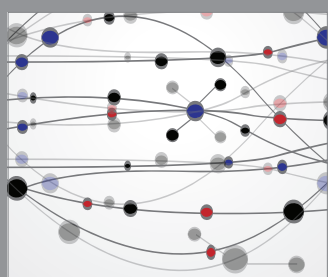

The Scientific World Journal

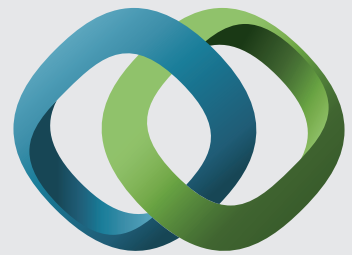

\section{Hindawi}

Submit your manuscripts at

http://www.hindawi.com
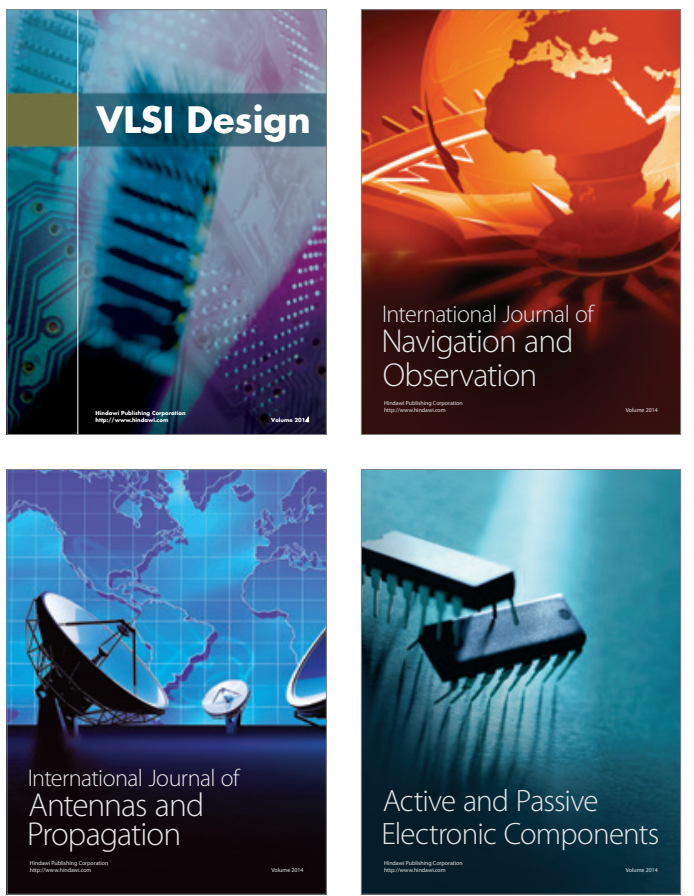
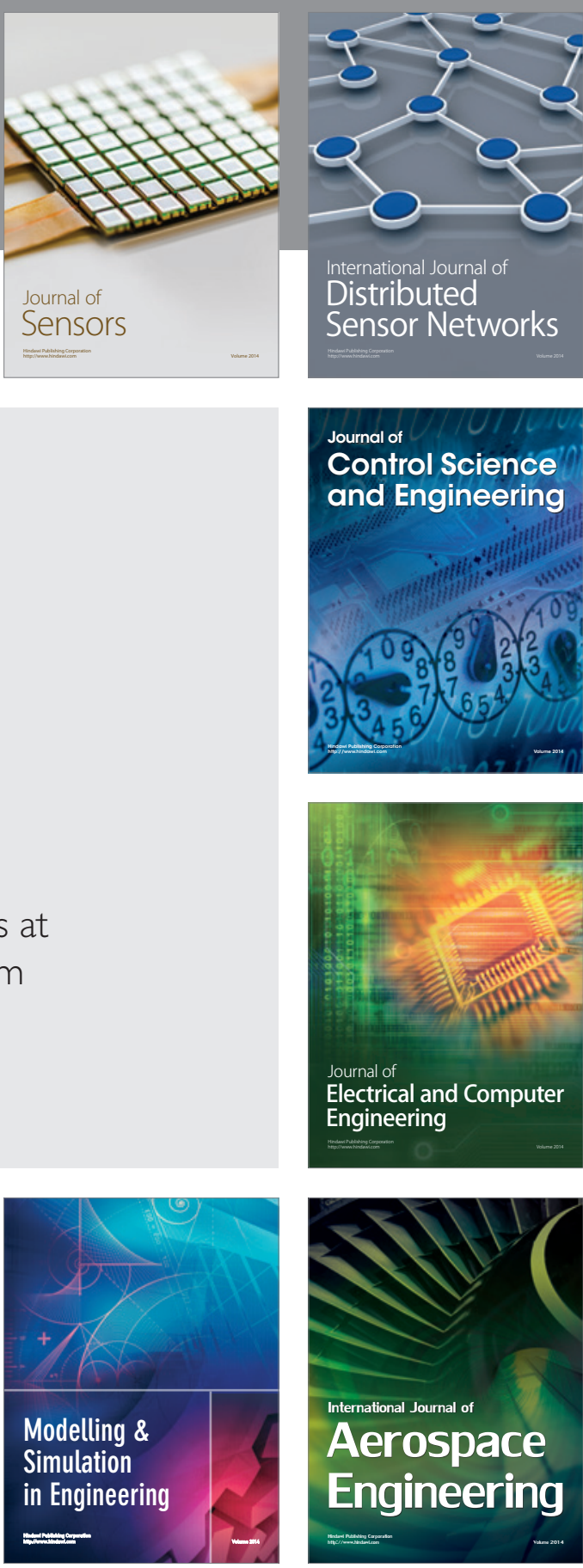

International Journal of

Distributed

Sensor Networks

Journal of

Control Science

and Engineering
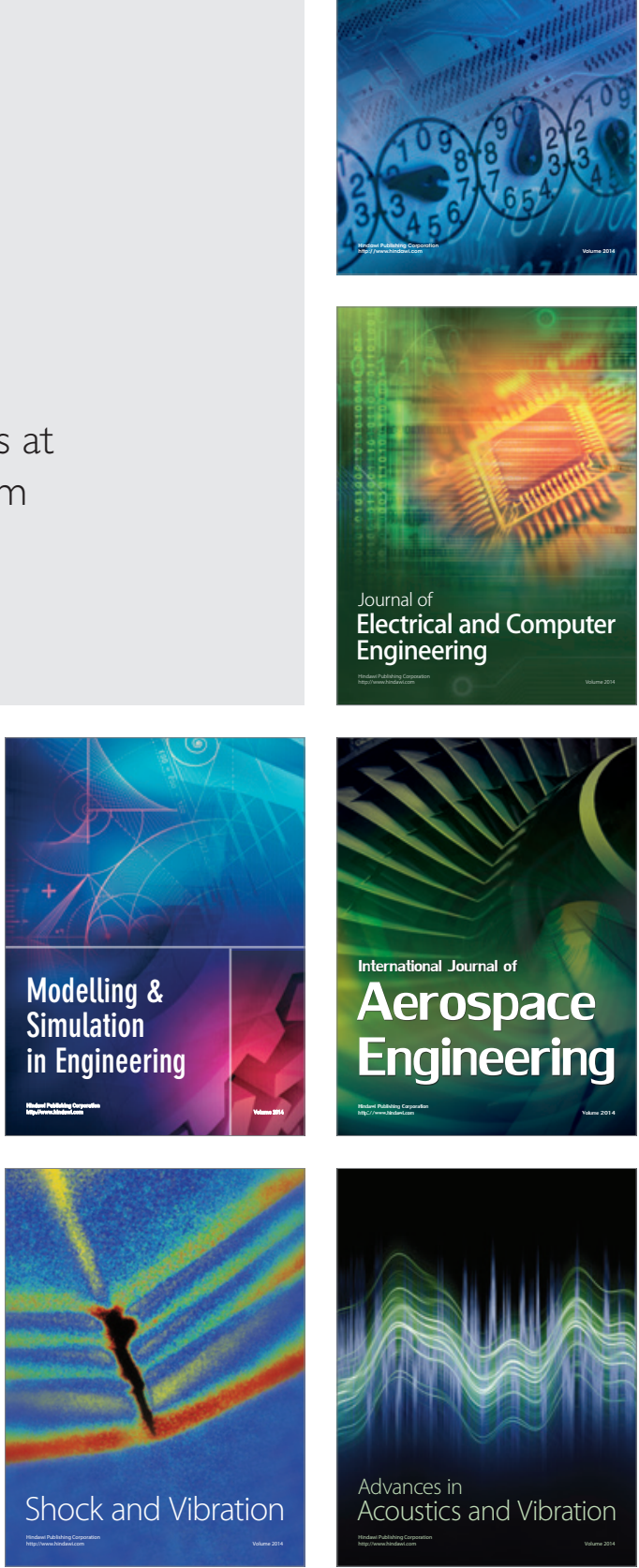